\title{
DENSITY OF ONE GRAPH ALONG ANOTHER ${ }^{1}$
}

JACK B. BROWN

Introduction. The word "graph" shall mean the graph of a real function, and the $X$-projection of a graph $F$ is the set of all abscissas of points of $F$. $c$ denotes the cardinality of the continuum. If $H$ is a collection of sets, $H^{*}$ denotes the union of all the sets in $H$.

Definition. Suppose $F$ and $G$ are graphs with $X$-projection $[0,1]$. The statement that $F$ is dense (c-dense) along $G$ means that if $[a, b]$ is a subinterval of $[0,1]$, then there is a point (are $c$-many points) of intersection of $F$ and $G$ with abscissa in $[a, b]$. In this paper, the following three theorems will be proved:

ThEOREM 1. If $F$ is a graph with $X$-projection $[0,1]$, then $F$ is dense along the graph of a function of Baire class 1 with domain $[0,1]$. However, there is a graph with $X$-projection $[0,1]$ which is not dense along the graph of any continuous function with domain $[0,1]$.

THEOREM 2. If $F$ is a graph with $X$-projection $[0,1]$, then $F$ is $c$-dense along the graph of a Lebesgue measurable function with domain $[0,1]$. However, there is a graph with $X$-projection $[0,1]$ which is not $c$-dense along the graph of any Baire function with domain $[0,1]$.

THEOREM 3. There exists a graph $F$ with $X$-projection $[0,1]$ which is $c$-dense along the graph of every Lebesgue measurable function with domain $[0,1]$.

Proofs. The author is indebted to the referee for the short proof of Theorem 1 which appears here. It makes use of a theorem of $\mathrm{H}$. Blumberg and is considerably shorter than the author's original proof.

Proof of Theorem 1. Suppose $F$ is the graph of a function $f$ with domain [0, 1]. It follows from Theorem III of [1] that there is a countable dense subset $D$ of $[0,1]$ such that $f \mid D$, the restriction of $f$ to $D$, is continuous. Now, if $f \mid D$ is bounded, let $g$ be defined as follows: $g(x)=f(x)$ if $x$ is in $D$, and if $x$ is in $[0,1]-D, g(x)$ is the $\lim \sup f(t)$ as $t \rightarrow x$ with $t$ in $D$. Then $g$ is upper semicontinuous and is therefore in Baire class 1 , and $F$ is dense along the graph of $g$. Now if $f \mid D$ is not bounded, let $s_{1}, s_{2}, \cdots$ be a sequence of mutually exclusive segments such that $D$ is a subset of $U s_{j}$ and $f \mid D$ is bounded on each $s_{j}$. For each

Presented to the Society, November 17, 1967; received by the editors September $20,1967$.

1 This work was supported by the U. S. Atomic Energy Commission. 
positive integer $j$, let $g_{j}$ be an upper semicontinuous function defined on $s_{j}$ which agrees with $f$ on $D \cap s_{j}$ and let $g$ be the function defined as follows: $g(x)=g_{j}(x)$ if $x$ is in $s_{j}$ and if $x$ is in $[0,1]-U s_{j}, g(x)=0$. The function $g$ is in Baire class 1 on the open set $U_{s_{j}}$ and on the closed set $[0,1]-U_{s_{j}}$. It is therefore in class 1 on all of $[0,1]$, and $F$ is dense along its graph.

The requirement of the second part of the theorem is satisfied by the graph of any function $f$ with domain $[0,1]$ such that for some $t$ in $(0,1), f(t+)$ and $f(t-)$ both exist but are unequal.

Proof of Theorem 2. Suppose $F$ is the graph of a function $f$ with domain $[0,1]$. Let $M$ be a subset of $[0,1]$ with measure zero such that if $[a, b]$ is a subinterval of $[0,1]$, then $[a, b] \cap M$ has cardinality $c$. Let $g(i)=f(t)$ if $t$ is in $M$ and $g(t)=0$ if $t$ is in $[0,1]-M$. Clearly, $g$ is Lebesgue measurable, and $F$ is $c$-dense along its graph.

Now, let $R$, the set of all real numbers, be well ordered so that no element of $R$ is preceded by $c$ elements, and let $T$ be a reversible transformation from $[0,1]$ to the class of all Baire functions with domain $[0,1]$. For each element $X$ of $[0,1]$, let $f_{X}$ denote $T(X)$. Let $h$ be the function defined as follows: for the first element $X$ of $[0,1], h(X)$ is the first element of $R$ different from $f_{X}(X)$, and if $Y$ is an element of $[0,1]$ such that $h(X)$ has been defined for every $X$ in $[0,1]$ which precedes $Y$, then $h(Y)$ is the first element of $R$ not in $\left\{f_{X}(Y) \mid X\right.$ is in $[0,1]$ and $X$ precedes $Y\}$. If $F$ is the graph of a Baire function with domain $[0,1]$ and $H$ is the graph of $h$, then the set of all points of intersection of $F$ and $H$ has cardinality less than $c$. Therefore $H$ is not $c$-dense along the graph of any Baire function with domain $[0,1]$.

Proof OF THEOREM 3. Every closed subset of $[0,1]$ which has positive measure has a perfect subset (the set of its points of condensation) and is therefore of cardinality $c$. The collection of all such sets is of cardinality $c$, so from Theorem 2, p. 458, of [2] it follows that there is a collection $W$ of $c$ mutually exclusive subsets of $[0,1]$, each of which has $c$ elements in common with each closed subset of $[0,1]$ of positive measure. Each set of $W$ has outer measure one, or else there would be a closed subset of $[0,1]$ of positive measure which some set of $W$ would not intersect. Let $T$ be a reversible transformation from $W$ to the class of all Baire functions with domain $[0,1]$ and for each set $w$ of $W$, let $g_{w}$ denote $T(w)$. Let $f$ be the function with domain $[0,1]$ defined as follows: if $t$ belongs to a set $w$ of $W, f(t)$ $=g_{w}(t)$, and if $t$ is in $[0,1]-W^{*}, f(t)=0$. Let $F$ be the graph of $f$. Suppose $G$ is the graph of a Lebesgue measurable function $g$ with domain $[0,1]$ and $[a, b]$ is a subinterval of $[0,1]$. Let $[d, e]$ be a proper subinterval of $[a, b]$ and $g^{\prime}$ be a Baire function with domain 
$[0,1]$ which agrees with $g$ except on a set $M$ of measure zero. Let $g^{\prime \prime}$ be that subset of $g^{\prime}$ which has domain $[d, e] . g^{\prime \prime}$ is a subset of $c$ different Baire functions $h$ with domain $[0,1]$, and for each of these a number $t_{h}$ in $[d, e]$ shall be chosen such that $f\left(t_{h}\right)=g\left(t_{h}\right)$. Let $h$ be a Baire function with domain $[0,1]$ of which $g^{\prime \prime}$ is a subset, and let $w$ be $T^{-1}(h)$. Since $w$ has outer measure one, there is a number $t_{h}$ which is in $w \cap[d, e]$ but not in $M$. Thus $f\left(t_{h}\right)=h\left(t_{h}\right)=g^{\prime \prime}\left(t_{h}\right)=g^{\prime}\left(t_{h}\right)=g\left(t_{h}\right)$. Since the sets in $W$ are mutually exclusive, no two numbers $t_{h}$ so chosen will be the same. Therefore, the set of points common to $F$ and $G$ which have abscissa in $[d, e]$ and therefore in $[a, b]$ has cardinality $c$.

\section{REFERENCES}

1. H. Blumberg, New properties of all real functions, Trans. Amer. Math. Soc. 24 (1922), 113-128.

2. W. Sierpinski, Cardinal and ordinal numbers, 2nd rev. ed., Polska Akad. Nauk, Monogr. Mat. 34, PWN-Polish Scientific Publishers, Warsaw, 1965.

\section{SANDia CoRporation and}

AUBURN UNIVERSITY 